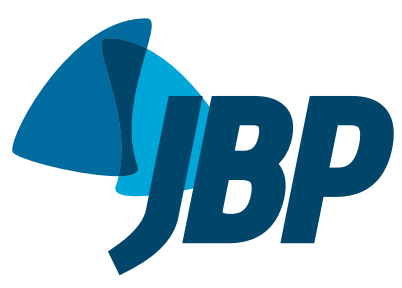

1. Serviço de Cirurgia Torácica, Pavilhão Pereira Filho, Irmandade da Santa Casa de Misericórdia de Porto Alegre, Porto Alegre (RS) Brasil.

2. Disciplina de Cirurgia Torácica, Universidade de Ciências da Saúde de Porto Alegre, Porto Alegre (RS) Brasil.

Submitted: 26 January 2016 Accepted: 31 October 2016.

Study carried out at the Serviço de Cirurgia Torácica, Pavilhão Pereira Filho, Irmandade da Santa Casa de Misericórdia de Porto Alegre, Porto Alegre (RS) Brasil.

\section{Anatomic pulmonary resection via video-assisted thoracic surgery: analysis of 117 cases at a referral center in Brazil}

\author{
Stephan Adamour Soder ${ }^{1}$, Frederico Barth ${ }^{1}$, Fabiola Adelia Perin ${ }^{1}$ \\ José Carlos Felicetti1,2, José de Jesus Peixoto Camargo, ${ }^{1,2}$ \\ Spencer Marcantônio Camargo ${ }^{1}$
}

\begin{abstract}
Objective: To describe our experience with video-assisted thoracic surgery (VATS) for anatomic pulmonary resection at a referral center for thoracic surgery in Brazil. Methods: All patients who underwent anatomic pulmonary resection by VATS between 2010 and 2015 were included. Clinical and pathological data, as well as postoperative complications, were analyzed. Results: A total of 117 pulmonary resections by VATS were performed, of which 98 were lobectomies and 19 were anatomic segmentectomies. The mean age of the patients was 63.6 years (range, $15-86$ years). Females predominated ( $n=69$; $59 \%)$. The mean time to chest tube removal was 2.47 days, and the mean length of ICU stay was 1.88 days. The mean length of hospital stay was 4.48 days. Bleeding $\geq$ $400 \mathrm{~mL}$ occurred in 15 patients. Conversion to thoracotomy was required in 4 patients. Conclusions: Our results are similar to those published in major international studies, indicating that VATS is an important strategy for pulmonary resection. They also show that VATS can be safely performed with adequate training. This technique should be used more often for the treatment of lung diseases in Brazil.
\end{abstract}

Keywords: Lung neoplasms/therapy; Lung neoplasms/complications; Thoracic surgery, video-assisted.

\section{INTRODUCTION}

Lung cancer is the leading cause of cancer death in Brazil and around the world. ${ }^{(1,2)}$ For 2014 in Brazil, 16,400 and 10,930 new cases of lung cancer were estimated to occur among men and women, respectively. These values correspond to an estimated risk of 16.79 new cases per 100,000 men and 10.75 new cases per 100,000 women. The latest global estimate was of an incidence of 1.82 million new cases of lung cancer in 2012, 1.24 million occurring in men and 583,000 occurring in women. ${ }^{(1)}$ Surgical treatment is the gold standard for early-stage neoplasms. First described in the early 1990's, video-assisted thoracic surgery (VATS) lobectomy has been increasingly used in daily practice for the treatment of lung cancer. Data from a Society of Thoracic Surgeons demonstrate that $44.7 \%$ of pulmonary resections in 2010 were performed via VATS. ${ }^{(3)}$

In addition to malignant neoplasms, other lung diseases might require surgical treatment, such as those of benign neoplastic, inflammatory, and infectious etiology, which can also be treated by VATS resection. -The potential documented benefits of VATS pulmonary resection include smaller incisions, lower pain intensity, a lower rate of ventilatory complications, minimal impact on the immune system, decreased release of inflammatory mediators, shorter hospital stays, etc. ${ }^{(3-6)}$

VATS should follow the sample principles as those of conventional surgery with anatomic pulmonary resection and mediastinal lymphadenectomy. -VATS provides good oncologic results, and various studies have demonstrated that the number of lymph nodes resected by VATS is similar to that of those resected by conventional surgery, ${ }^{(7-9)}$ without compromise of oncologic results. ${ }^{(10-12)}$ Survival results for patients undergoing VATS lobectomy are similar to those for patients undergoing conventional lobectomy, and 5-year disease-free survival is up to $88 \%$ in stage IA patients. ${ }^{(10)}$

Although VATS is a well-established technique worldwide, it is not yet part of routine practice in many thoracic surgery centers in Brazil. The poor availability of materials and equipment essential for performing these procedures, both in the public and private health care systems, has delayed this technique's introduction to and consolidation in many health care facilities in the country.

\section{METHODS}

\section{Patient selection and statistical analysis}

The objective of the present study was to determine the clinical and epidemiological profile of patients who underwent anatomic pulmonary resection by VATS in the Department of Thoracic Surgery of the Santa Casa Sisters of Mercy Hospital Pavilhão Pereira Filho, located in the city of Porto Alegre, Brazil. All patients who underwent anatomic pulmonary resection by VATS from 2010, when the first such procedure was performed, to 2015 were 
analyzed. In the present study, the following variables were assessed: gender; clinical stage; histological type; tumor location; type of resection; and relevant postoperative data. All data were collected prospectively and analyzed.

Categorical variables were calculated as frequencies. Continuous numerical variables were calculated as means; however, medians and standard deviations were also calculated, because some parameters exhibited non-normal data distribution and a wide data range. All statistical analyses were performed with IBM SPSS Statistics, version 22 (IBM Corporation, Armonk, NY, USA).

\section{Preoperative assessment}

All patients with suspected or confirmed lung cancer who were candidates for VATS resection underwent CT scanning of the chest and upper abdomen before surgery. Positron emission tomography/CT was performed whenever available, because there is a directive regulating the use of this test in the public health care system, and private health insurance companies also have specific criteria for its use. Pulmonary function was assessed by spirometry. Staging was performed in accordance with the recommendations of major guidelines for preoperative assessment and management of lung cancer, especially those of American ${ }^{(13)}$ and European $^{(14)}$ guidelines.

\section{Surgical technique}

Patients underwent general anesthesia with elective intubation and were placed in the lateral decubitus position. The surgical technique used was that described by a group from Duke University, ${ }^{(15)}$ consisting of placement of a $10-\mathrm{mm}$ trocar for placement of video optics in the 8th intercostal space, near the midaxillary line. A working port was placed in the 4th or 5th intercostal space, by means of a $4-$ to $6-\mathrm{cm}$ incision crossing the anterior axillary line. In some cases, a third port was used for introducing auxiliary forceps and vascular staplers, being usually placed in the 8th intercostal space close to the posterior axillary line, as shown in Figure 1. Bronchial and vascular ligation was performed with a thoracoscopic linear stapler. Mediastinal lymphadenectomy was performed in all cases of neoplastic disease.

\section{RESULTS}

In the study period, a total of 117 VATS pulmonary resections were performed, of which 98 were lobectomies and 19 were anatomic segmentectomies. The study population was mostly composed of female patients (59\%). The median age was 65 years (range, 15-86 years), and the mean age was $63.6 \pm 13.2$ years. Approximately one fourth $(26.5 \%)$ of the patients had no history of smoking. Among smokers, the mean smoking history was 44.2 pack-years. The resection profile is shown in Table 1.

The median intraoperative bleeding was $100 \mathrm{~mL}$ (mean, $189 \pm 260 \mathrm{~mL}$ ), and bleeding less than or equal to $100 \mathrm{~mL}$ occurred in 69 patients (59\%). Intraoperative bleeding greater than or equal to 400 $\mathrm{mL}$ occurred in 10 patients $(8.5 \%)$, being mostly secondary to the dissection itself and the handling of small vessels, with smaller lesions being successfully managed by VATS. Arterial vascular injury requiring transfusion of blood components and conversion to thoracotomy occurred in 2 patients, but without any major hemodynamic instability.

The mean time to chest tube removal was $2.47 \pm$ 2.18 days (median, 2 days), and the mean length of

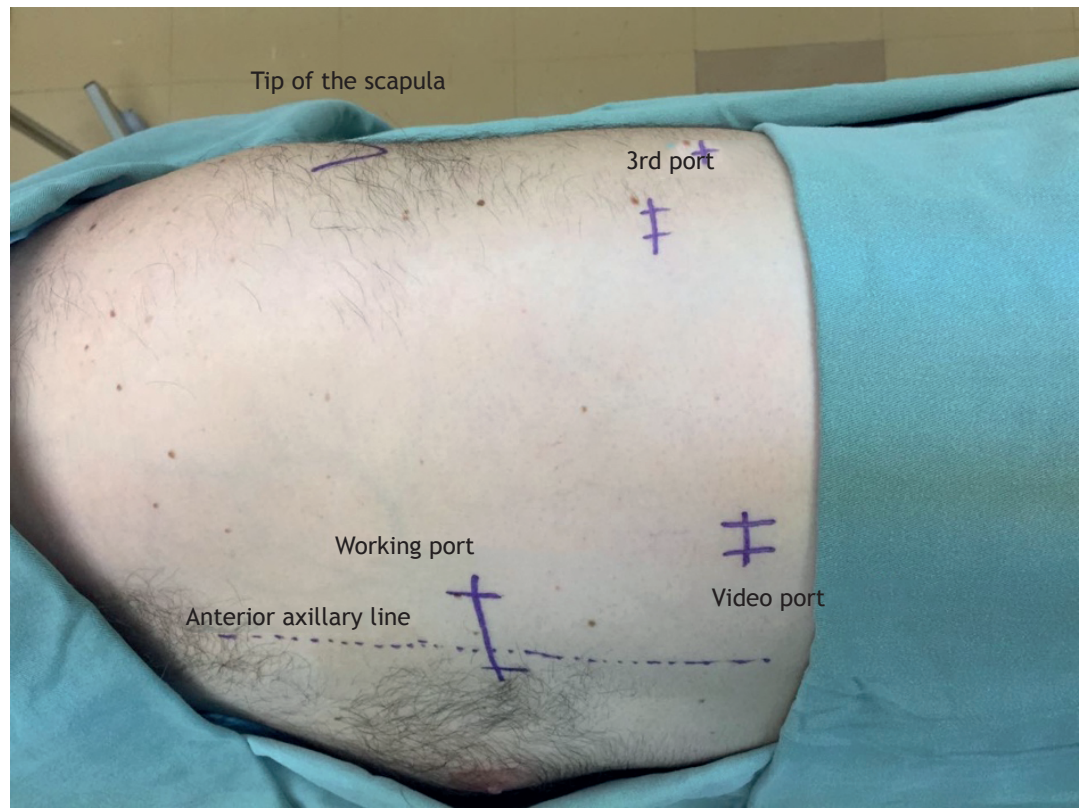

Figure 1. Anatomical representation of the surgical technique used. 
ICU stay was $1.88 \pm 1.88$ days (median, 2 days). The mean length of hospital stay was $4.48 \pm 3.54$ days (median, 4 days). Some patients remained in the hospital after chest tube removal for clinical optimization. In this series, conversion to the open technique was required in 4 patients $(3.4 \%)$, because of bleeding, in 2 ; because bronchoplasty was necessary, in 1 ; and because of a technical intraoperative decision, in 1. Although in these last two cases, imaging and endoscopic findings had shown that the lesions were central in location, VATS was performed, and the surgery proceeded as far as feasible. Postoperative complications included 6 cases of complicated pleural effusion and empyema, all of which were treated by pleuroscopy; 1 case of middle lobe torsion, which was treated by VATS middle lobectomy; and 1 case of prolonged air leak, in which a small thoracotomy was performed to treat the air fistula. There was 1 case of death within the first 30 days after surgery, resulting from complications from empyema and sepsis. There were no cases of intraoperative death.

Perioperative morbidity included 5 cases of empyema; 4 cases of prolonged chest tube air leak (defined as an air leak lasting more than 7 days); 4 cases of postoperative delirium; 2 cases of acute renal failure requiring dialytic therapy; 2 cases of atrial fibrillation with rapid ventricular response; 2 cases of pneumonia; and 1 case of pneumothorax and delayed subcutaneous emphysema.

As shown in Table 2, the etiology of most of the lesions treated in our study sample was malignant neoplasm (83.7\% of the cases), with 87 resections for primary lung cancer, in which the predominant histological type was adenocarcinoma, and 11 cases of lung metastasis, 6 of which resulted from lesions that were colonic in origin, and with the remaining cases being of metastasis of sarcoma, melanoma, and clear cell renal carcinoma. Nineteen patients underwent anatomic pulmonary resection for the treatment of non-neoplastic diseases, the vast majority of whom had bronchiectasis, and 1 of those patients underwent the procedure for the treatment of fungal disease sequelae.

Table 1. Resection by lobectomy $(n=98)$ or by segmentectomy $(n=19)$.

\begin{tabular}{lc}
\multicolumn{1}{c}{ Lobectomy } & $\mathbf{n}(\%)$ \\
\hline Right upper & $19(19.4)$ \\
Middle & $6(6.1)$ \\
Right lower & $20(20.4)$ \\
Left upper & $26(26.5)$ \\
Left lower & $26(26.5)$ \\
Bilobectomy & $1(1.0)$ \\
\hline \multicolumn{1}{c}{ Segmentectomy } & \\
Culmen & $5(26.3)$ \\
Lingula & $4(21.1)$ \\
Basal pyramid & $2(10.5)$ \\
Upper segment & $5(26.3)$ \\
Lower segment & $2(10.5)$ \\
Bisegmentectomy & $1(5.3)$
\end{tabular}

Analysis of pathological staging as per the 7th edition of the tumor-node-metastasis (TNM) classification ${ }^{(16)}$ revealed that, in our sample, the vast majority of the malignant neoplastic lesions were stage I. Four patients had mediastinal lymph nodes that were affected by cancer and required adjuvant therapy. Table 3 specifies the number of lesions secondary to non-small cell tumors, according to the TNM pathological staging classification.

\section{DISCUSSION}

VATS pulmonary resection has increasingly become a reality in the treatment of patients in major centers for thoracic surgery in Brazil. In most of the 117 cases presented here, the procedures were performed in the last 2 years of the study period, after VATS was included by the Brazilian National Health Insurance Agency in the list of operations covered by health insurance plans. The growing experience in performing VATS and mastering the technique has made it possible to expand its use into the routine surgical treatment of lung diseases. Between January and September of 2015 , approximately $30 \%$ of lobectomies at the Pavilhão Pereira Filho were performed via VATS.

One of the major concerns regarding VATS, especially in the stages of method introduction and at resident training centers, is the occurrence of bleeding and the forms of controlling it once it has occurred. We have observed that proper and careful handling of vessels and fissures makes it possible to perform these operations with low bleeding volume, even at resident training centers. In the present series, there were only 10 procedures in which intraoperative bleeding was estimated at more than $400 \mathrm{~mL}$ and

Table 2. Etiology (histological type) of the 117 cases included in the study.

\section{Etiology (histological type)}

n $(\%)$

Adenocarcinoma $73(62.4)$

Epidermoid carcinoma

$09(7.7)$

Large cell neuroendocrine tumor

$01(0.9)$

Small cell neuroendocrine tumor

Carcinoid tumor

Metastases

$11(9.4)$

Inflammatory lesions

$19(16.3)$

Table 3. Stage of malignant lesions (non-small cell tumors; $\mathrm{N}=86$ ).

\begin{tabular}{cccc}
$\begin{array}{c}\text { TNM } \\
\text { classification }\end{array}$ & $\mathbf{n}(\%)$ & $\begin{array}{c}\text { Pathological } \\
\text { stage }\end{array}$ & $\mathbf{n}(\%)$ \\
T1aN0M0 & $21(24.4)$ & IA & $30(34.9)$ \\
T1bN0M0 & $9(10.5)$ & IB & $41(47.7)$ \\
T2aNOM0 & $41(47.7)$ & IIA & $10(11.6)$ \\
T2bN0M0 & $3(3.5)$ & IIB & $1(1.2)$ \\
T1bN1M0 & $1(1.2)$ & IIIA & $4(4.7)$ \\
T2aN1M0 & $6(7.0)$ & & \\
T3N0M0 & $1(1.2)$ & & \\
T2aN2M0 & $4(4.7)$ & & \\
\hline
\end{tabular}

TNM: tumor-node-metastasis. 
two conversions to thoracotomy for bleeding control, without that implying any technical compromise or affecting patient treatment.

The morbidity and mortality results obtained in our initial 5-year experience were found to be comparable to those reported in major international series. McKenna et al. published a large series of 1,100 cases in $2006,{ }^{(6)}$ reporting low rates of postoperative complications and demonstrating the feasibility and effectiveness of VATS lobectomy at a center experienced in this method. In their series, they found a conversion rate of $2.5 \%$ and a postoperative mortality rate of $0.8 \%$. In 2008 , Nicastri et al. ${ }^{(5)}$ also reported a postoperative mortality rate of $0.7 \%$, although their reported rate of conversion to thoracotomy was slightly higher (9.2\%). In a literature review of more than 6,000 cases, Whitson et al. ${ }^{(11)}$ found shorter time to chest tube removal, shorter hospital stays, and lower rates of postoperative complications in patients who underwent VATS resection than in those who underwent thoracotomy resection.

One of the objectives of VATS is to reduce the need for invasive management of patients, avoiding the use of neuraxial catheters for analgesia and the use of probes, which also reduces the need for ICU admission. The Pavilhão Pereira Filho has a dedicated surgical ICU. Although most of our patients did not require epidural analgesia, the routine of a 24-hour postoperative recovery period in the ICU was maintained for all patients. This explains, in part, the mean length of ICU stay of 1.88 days. In the present series, the median length of hospital stay was 4 days, with a mean of 4.48 days. In the future, with increased experience, we intend to reduce the length of patient stay in the ICU.

Although assessment of the amount of analgesia used was not one of our objectives, we found that there was a clear reduction in pain reporting by patients, which is much more evident at the first outpatient follow-up visit, when most patients return to their usual activities. Although we did not use a pain score, the continuity of the experience in the present sample allowed us to dispense with placing epidural catheters in patients, which were used in some of the first cases but were not longer used in the last half of the series, translating to an important objective datum on pain reduction.

When analyzing the group of sublobar resections (19 cases) only, we observed that $36.8 \%$ were performed for the treatment of primary neoplasms, $26.4 \%$ for the treatment of metastases, and $36.8 \%$ for the treatment of diseases with an inflammatory etiology. Although lobar resection remains the gold standard in the treatment of lung carcinoma, recent studies have demonstrated that anatomic segmentectomy with attention to adequate surgical margins and lymph node dissection may have results equivalent to those reported for lobectomy, ${ }^{(17)}$ being considered a reasonable oncologic option particularly in patients with impaired pulmonary function who may not tolerate a lobectomy.

The present study lacks the power to estimate patient survival accurately, given that most resections were performed in the last 2 years. We intend to publish such data when the follow-up period is longer.

VATS pulmonary resection is being introduced in many centers in Brazil and is already part of routine practice in some. The present study sought to demonstrate that this technique can be safely performed and can provide good results, even at resident training centers. In the future, more studies will demonstrate the impact of VATS on the medium- and long-term oncologic results in the population undergoing such procedures in Brazil.

\section{REFERENCES}

1. Instituto Nacional de Câncer José Alencar Gomes da Silva Coordenação de Prevenção e Vigilância. Estimativa 2014: Incidência de Câncer no Brasil. Rio de Janeiro: INCA; 2014

2. American Cancer Society. Cancer Facts \& Figures 2015. Atlanta, GA American Cancer Society; 2015

3. Ceppa DP, Kosinski AS, Berry MF, Tong BC, Harpole DH, Mitchell JD, et al. Thoracoscopic lobectomy has increasing benefit in patients with poor pulmonary function: a Society of Thoracic Surgeons Database analysis. Ann Surg 2012;256(3):487-93. https://doi. org/10.1097/SLA.0b013e318265819c

4. Swanson SJ, Meyers BF, Gunnarsson CL, Moore M, Howington JA, Maddaus MA, et al. Video-assisted thoracoscopic lobectomy is less costly and morbid than open lobectomy: a retrospective multiinstitutional databaseanalysis. Ann Thorac Surg. 2012;93(4):102732. https://doi.org/10.1016/j.athoracsur.2011.06.007

5. Nicastri DG, Wisnivesky JP, Litle VR, Yun J, Chin C, Dembitze $F R$, et al. Thoracoscopic lobectomy: report on safety, discharge independence, pain, and chemotherapy tolerance. J Thorac Cardiovasc Surg. 2008;135(3):642-7. https://doi.org/10.1016/j. jtcvs.2007.09.014

6. McKenna RJ Jr, Houck W, Fuller CB. Video-assisted thoracic surgery lobectomy: experience with 1,100 cases. Ann Thorac Surg. 2006;81(2):421-5; discussion 425-6. https://doi.org/10.1016/j athoracsur.2005.07.078

7. Kondo T, Sagawa M, Tanita T, Sato M, Ono S, Matsumura Y, et al. Is complete systematic nodal dissection by thoracoscopic surgery possible? A prospective trial of video-assisted lobectomy for cancer

of the right lung. J Thorac Cardiovasc Surg. 1998;116(4):651-2 https://doi.org/10.1016/S0022-5223(98)70175-8

8. Sagawa M, Sato M, Sakurada A, Matsumura Y, Endo C, Handa M, et al. A prospective trial of systematic nodal dissection for lung cancer by video-assisted thoracic surgery: can it be perfect? Ann Thorac Surg 2002;73(3):900-4. https://doi.org/10.1016/S0003-4975(01)03409-9

9. McKenna RJ Jr. Lobectomy by video-assisted thoracic surgery with mediastinal node sampling for lung cancer. J Thorac Cardiovasc Surg. 1994; 107(3):879-81; discussion 881-2

10. Higuchi M, Yaginuma $H$, Yonechi A, Kanno R, Ohishi A, Suzuki H, et al. Long-term outcomes after video-assisted thoracic surgery (VATS) lobectomy versus lobectomy via open thoracotomy for clinical stage IA non-small cell lung cancer. J Cardiothorac Surg. 2014;9:88. https:// doi.org/10.1186/1749-8090-9-88

11. Whitson BA, Groth SS, Duval SJ, Swanson SJ, Maddaus MA. Surgery for early-stage non-small cell lung cancer: a systematic review of the video-assisted thoracoscopic surgery versus thoracotomy approaches to lobectomy. Ann Thorac Surg. 2008,86(6):2008-16 discussion 2016-8

12. Yan TD, Black D, Bannon PG, McCaughan BC. Systematic review and meta-analysis of randomized and nonrandomized trials on safety and efficacy of video-assisted thoracic surgery lobectomy for earlystage non-small cell lung cancer. J Clin Oncol. 2009;27(15):2553-62. https://doi.org/10.1200/JCO.2008.18.2733

13. Silvestri GA, Gonzalez AV, Jantz MA, Margolis ML, Gould MK Tanoue LT, et al. Methods for staging non-small cell lung cancer: Diagnosis and management of lung cancer, 3rd ed. American College 
of Chest Physicians evidence-based clinical practice guidelines. Chest. 2013;143(5 Suppl):e211S-50S

14. De Leyn P, Dooms C, Kuzdzal J, Lardinois D, Passlick B, Rami-Porta $R$, et al. Revised ESTS guidelines for preoperative mediastinal lymph node staging for non-small-cell lung cancer. Eur J Cardiothorac Surg. 2014;45(5):787-98. https://doi.org/10.1093/ejcts/ezu028

15. D'Amico TA. Operative techniques in early-stage lung cancer. J Natl Compr Canc Netw. 2010,8(7):807-13.
16. Lung. In: Edge SB, Byrd DR, Compton CC, Fritz AG, Greene FL, Trotti A, editors. AJCC Cancer Staging Manual. 7th ed. New York, NY: Springer; 2010. p. 253-70. https://doi.org/10.1007/978-0-38788441-7_25

17. Altorki NK, Yip R, Hanaoka T, Bauer T, Aye R, Kohman L, etal. Sublobar resection is equivalent to lobectomy for clinical stage $1 \mathrm{~A}$ lung cancer in solid nodules. J Thorac Cardiovasc Surg. 2014;147(2):754-62; Discussion 762-4. https://doi.org/10.1016/j.jtcvs.2013.09.065 